\title{
Simulación de una red empresarial mediante la herramienta Cisco Packet Tracer
}

Fecha de recepción: 2021-07-26 Fecha de aceptación: 2021-09-15 • Fecha de publicación: 2021-10-10

Carlos Alexander Chicaiza Puedmag

Universidad Central del Ecuador

cachicaizap@gmail.com

https://orcid.org/0000-0003-0141-4654

\section{RESUMEN}

A partir de la pandemia suscitada en el año 2020 se han tenido que postergar varias actividades de diferentes sectores, mientras que otras han migrado de la modalidad presencial a la virtual. La educación es una de estas que ha tenido que cambiar, aunque en un inicio fue complicado adaptarse a la modalidad online, muchas aplicaciones han ayudado en este proceso, estas aplicaciones, de hecho, han llegado a tener un mayor auge a causa de ello, una de esas plataformas es Cisco Packet Tracer. Dicho programa mencionado anteriormente permite realizar simulaciones de redes empresariales, ya que, dentro de la plataforma se pueden utilizar distintos dispositivos de hardware, al igual que permite realizar la configuración de cada uno de esos dispositivos, por lo cual, la herramienta es de gran importancia, tanto para las universidades, como para los estudiantes, ya que permite estudiar a mayor profundidad cada uno de los dispositivos de red que emula la aplicación.

\section{PALABRAS CLAVE: cisco, hardware, dispositivos, red, educación virtual.}

\section{ABSTRACT}

Since the pandemic in 2020, several activities in different sectors have had to be postponed, while others have migrated from face-to-face to virtual mode. Education is one of these that has had to 
change, although at first it was complicated to adapt to the online mode, many applications have helped in this process, these applications, in fact, have come to have a greater boom because of it, one of these platforms is Cisco Packet Tracer. The aforementioned program allows you to perform simulations of enterprise networks, since, within the platform you can use different hardware devices, as well as allowing the configuration of each of these devices, which is why the tool is of great importance, both for universities and for students, as it allows to study in greater depth each of the network devices that emulates the application.

KEYWORDS: cisco, hardware, devices, red, education, virtual. 


\section{Introducción}

Dentro de la nueva normalidad que se presenta no solo en este, sino en varios países, se ha tenido que cambiar un aula de clases por una habitación del lugar donde se vive, perdiendo el acceso a varios dispositivos que permitían aprender, cambiándolos por un computador y un sinfín de aplicaciones que permitan el acceso a la educación. Una de las cosas que se perdieron con el cambio de ambiente universitario, en el caso de algunas carreras técnicas y de ingeniería, fue la posibilidad de poder trabajar y configurar, de forma presencial, algunos dispositivos de red.

No obstante, una de las cosas que se necesita aprender durante las carreras mencionadas, es la utilización de dispositivos de red, y el montaje y configuración de una red como tal. Pero, al estar en un ambiente virtual, no existe otro camino más que simular un ambiente real y hacer las configuraciones sobre plataformas virtuales que les permitan aplicar algunos de los conocimientos teóricos en la práctica.

Dentro de una empresa, las redes son de gran importancia, ya que mediante estas se puede compartir información, recursos y servicios entre distintos usuarios, no solo de una forma ágil, sino también segura. Por tanto, las compañías, ya sean públicas o privadas, intentan implementar correctamente redes físicas en puntos específicos que les permita que el flujo de información entre usuarios sea veloz, y al mismo tiempo, poder administrar y organizar tanto los recursos de un departamento, como a los usuarios de este.

Ahora bien, por lo anterior, se puede decir que es de gran importancia que los estudiantes puedan no solo entender qué es una red, sino también simular una, y esto es lo que pretende este artículo, simulará el proceso de configuración y construcción de una red LAN de comunicación, por lo cual se utilizarán varias herramientas como lo serán computadoras, switch, router, access point, cámara, entre otros instrumentos de hardware y de software que permitirán levantar dicha red.

Si bien el proceso de diseño, construcción y configuración de una red es más grande de lo que se presentará a continuación, el desarrollo que será expuesto en este artículo permitirá visualizar y entender de mejor manera el funcionamiento e importancia que tiene una red dentro de una institución.

Para la siguiente simulación de red, se usa el software Cisco Packet Tracer, mediante el cual se realiza la configuración de dispositivos como si se los manipulará de forma real. Cisco Packet Tracer permite realizar simulaciones en tiempo real, al igual que verifica el recorrido que tiene un paquete dentro de la red, de esta manera se comprueba que la red es funcional. En esta plataforma se pueden crear conexiones básicas, como lo es la conexión entre dos computadores e incluso conexiones más complejas, es decir, el plantear una red específica (Barragán \& Jurado, 2012).

Cisco Packet Tracer proporciona una amplia gama de dispositivos que van desde switches y routers, dispositivos inalámbricos como laptops, access point, hasta computadoras de escritorio y servidores. También se puede comprobar el funcionamiento de una red y los cambios que se van 
produciendo en la misma, al igual que posee una herramienta de evaluación en la cual se pueden crear preguntas prácticas sobre redes complejas (Jesin, 2014).

Este proyecto es realizado con el fin de demostrar que el uso de herramientas digitales puede ayudar a estudiantes a entender de mejor manera la utilización de equipos, que no están a su alcance, además, es muy importante que alumnos de tecnología aprendan a diseñar y configurar equipos, ya que les ayudará en su vida profesional. De lo dicho anteriormente surge la necesidad de que estos aprendan y se capaciten de forma teórica y experimental, es allí donde tiene sentido el utilizar un software correspondiente que permita emular el uso de distintos equipos físicos (Cevallos, 2010).

A continuación, en la Figura 1 se presenta un flujograma de las actividades que se realizarán dentro en la siguiente parte del artículo.

\section{Figura 1}

Flujograma de los procesos a realizar

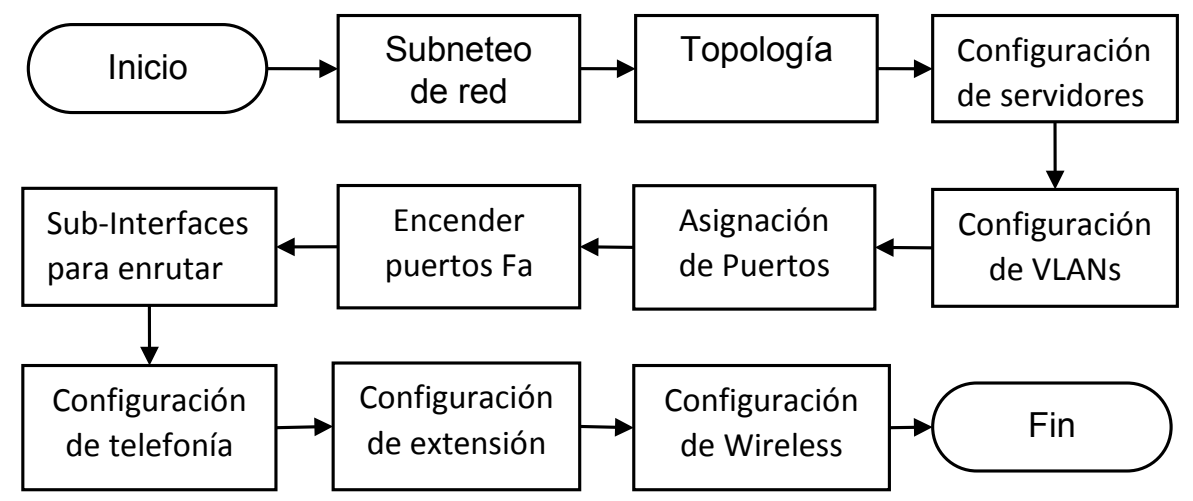

\section{Metodología}

En este punto se detalla el proceso de construcción de una red empresarial, pero antes de continuar, se definirán algunos conceptos que permitirán comprender de mejor manera el proceso de desarrollo de esta simulación.

Una red es un conjunto de más de dos computadoras que se encuentra conectadas entre sí, esta conexión puede ser física y/o lógica a través de los cuales se pueden compartir recursos, estos pueden ser de hardware o software. Esto permite a los usuarios utilizar cualquiera de los recursos mencionados desde cualquier lugar, sin necesitar que estén físicamente en su área de trabajo (Robleno, 1999).

Sin embargo, aunque ya se conoce el concepto de una red de comunicación y su funcionalidad, también es necesario conocer la topología de las redes de comunicación. 
- Red personal: está centrada en el usuario y designa una interconexión de equipos informáticos en un espacio de una decena de metros entorno al usuario (Dordoigne, 2015).

- Red local: es una red que vincula computadoras que se hallan en un espacio físico pequeño, como una oficina o un edificio. Gracias a la red, los usuarios de estas computadoras pueden compartir documentos e incluso hacer un uso común de ciertos periféricos. (Pérez Porto \& Gardey, 2015).

- Red metropolitana: garantiza la interconexión a distancias más extensas que una red LAN, es decir, realiza la interconexión de varias redes LAN. Un ejemplo de ello es que puede interconectar varios departamentos de una empresa que se encuentran demasiado lejos (Dordoigne, 2015).

- Red extendida: una red WAN es un tipo de red de computadoras capaz de cubrir distancias desde unos 100 hasta unos 1000 km, proveyendo de servicio a una ciudad, país o incluso a un continente (Kurose \& Ross,2006).

Por parte está el diseño jerárquico de una red, este consiste en la división de la red en capas independientes, con el objetivo de diseñar una infraestructura de red confiable, escalable y de alta disponibilidad. El diseño jerárquico de red o también conocido como 3 capas, se lo denomina así porque este compuesto por 3 capas como lo son la capa de núcleo, distribución y acceso, aunque no siempre se plantea el diseño en tres capas, ya que existe el núcleo contraído donde la capa de distribución y núcleo están juntas, y el de 3 niveles, donde las capas están separadas (Huang, 2017).

- Capa núcleo: es la que proporcionará un transporte rápido entre routers, también de lograr confiabilidad, y la capacidad de tolerar fallos al igual que poder ser escalable con equipos más rápidos (Gómez \& Pisco, 2018).

- Capa de distribución: establece una conexión entre las capas de núcleo y acceso, controlando el tráfico que circula entre ellas, permite configurar políticas de seguridad, para proteger la información dentro de la red (Gómez \& Pisco, 2018).

- Capa de acceso: como su nombre lo indica, proporciona un punto de acceso a los dispositivos finales, es la capa encargada de la conmutación y se conecta directamente con la capa de distribución (Gómez \& Pisco, 2018).

A continuación, se presentan los conceptos de algunos dispositivos que se utilizarán:

- Switch: es un dispositivo que permite que la conexión de computadoras y periféricos a la red, para que puedan comunicarse entre sí y con otras redes. Actúa como un controlador que permite que diferentes dispositivos compartan información entre sí (Significados, 2017).

- Access point: son dispositivos para establecer una conexión inalámbrica entre equipos y pueden formar una red inalámbrica externa (local o Internet) con la que interconectar dispositivos móviles o tarjetas de red inalámbricas. Esta red inalámbrica se llama WLAN (Wireless local área network) y se usan para reducir las conexiones cableadas (lawebdelprogramador, s.f). 
- Telefonía IP: es la telefonía que establece las comunicaciones mediante Internet, y donde la transición de voz se realiza mediante IP. La telefonía IP se está transformando en la forma de comunicación más utilizada por las empresas, sustituyendo los sistemas telefónicos tradicionales. Usa «protocolos de Internet» para comunicarse por medios digitales. Al usar esta tecnología, en vez de los sistemas telefónicos tradicionales, la empresa o el usuario puede aprovechar su conexión a Internet, el hardware y las aplicaciones para comunicarse de forma más eficaz (Citelia, s.f).

- Router: es un dispositivo que opera en capa tres de nivel de 3. Así, permite que varias redes u ordenadores se conecten entre sí y, por ejemplo, compartan una misma conexión de Internet. Un router se vale de un protocolo de enrutamiento, que le permite comunicarse con otros enrutadores o encaminadores y compartir información entre sí para saber cuál es la ruta más rápida y adecuada para enviar datos (Bembibre, 2009).

- Vlan: permite crear redes lógicamente independientes dentro de la misma red física, haciendo uso de switches gestionables que soporten VLANs para segmentar adecuadamente la red. También es muy importante que los routers que se utilicen soporten VLAN, de lo contrario no se podrá gestionar todas, ni permitir o denegar la comunicación entre ellas (De luz, 2021).

Por otra parte, es importante mencionar que una subred es un conjunto de dispositivos que comparten la misma dirección de subred y son gestionados por un mismo gestor de subred (Cámara, 2016).

Las subredes aumentan el control del manejo de la red en el espacio de dirección y proveen un mecanismo para usar routers cuando solamente uno o un número pequeño de números de redes está disponible (Donoso et al., 2001).

A su vez, una subneteo de red hace referencia a la subdivisión de una red en varias subredes. El subneteo permite a los administradores de red, por ejemplo, dividir una red empresarial en varias subredes sin hacerlo público en Internet. Esto se traduce en que el router que establece la conexión entre la red e Internet se especifica como dirección única, aunque puede que haya varios hosts ocultos. Así, el número de hosts que están a disposición del administrador aumenta considerablemente.

Los motivos para el subneteo de redes son múltiples. Las subredes funcionan de manera independiente las unas de las otras y la recogida de los datos se lleva a cabo con mayor celeridad (Godoy, 2018).

Las clases de redes que existen para nuestro uso, son 3:

- Clase A: Desde 0.0.0.0 hasta 127.255.255.255

- Clase B: Desde 128.0.0.0 hasta 191.255.255.255

- Clase C: Desde 192.0.0.0 hasta 223.255.255.255 
Las clases de máscaras de subred que tienen por default cada una de las clases de red son:

- Clase A: 255.0.0.0

- Clase B: 255.255.0.0

- Clase C: 255.255.255.0 (Donoso et al., 2001).

Después de dar algunos conceptos de los dispositivos que se utilizarán durante la simulación, se procederá a realizar la topología de la red, ya que se necesitará dividir a la red que ingresa en varias subredes, y montar un edificio principal, y un secundario o sucursal.

Para el subneteo de red se utilizará una IP de Clase C que será 192.168.1.0 y la máscara de subred será 255.255.255.0, ahora bien, se iniciará el proceso de subneteo de la red.

Se obtienen en total 16 subredes, de las cuales se van a utilizar para la practica 5 subredes. Se calculan los bits necesarios para la máscara adaptada, en las siguientes Tabla 1 y Tabla 2 se muestran.

Tabla 1

Máscara original clase $C$

\begin{tabular}{|c|c|c|c|}
\hline 255 & 255 & 255 & 0 \\
\hline 11111111 & 11111111 & 11111111 & 00000000 \\
\hline
\end{tabular}

Tabla 2

Máscara adaptada

\begin{tabular}{|c|c|c|c|}
\hline 255 & 255 & 255 & 240 \\
\hline 11111111 & 11111111 & 11111111 & 11110000 \\
\hline
\end{tabular}

Ahora se tienen que obtener los hosts, por lo cual se va a trabajar con la dirección IP de red, específicamente con la porción de host:

Se usa $2^{\mathrm{n}}-\mathbf{2}$ donde $\mathrm{n}$ es el número de bits disponibles en la porción de host. Se utiliza $\mathbf{- 2}$ porque la primera y la última dirección IP de la subred no se utilizan por ser la dirección de la subred y broadcast respectivamente.

Al aplicar la formula $2^{\mathrm{n}}-2=$ Host, donde $\mathrm{n}$ es la cantidad disponible en la parte del host, entonces $\boldsymbol{n}=4$. Por lo tanto, cada subred va a tener 14 host disponibles.

La forma más sencilla para obtener el rango es restarle a 256 el número de la máscara de subred adaptada. En este caso sería $\mathbf{2 5 6}$ - $\mathbf{2 4 0}=\mathbf{1 6}$, entonces 16 va a ser el rango entre cada subred. Por lo anterior tendremos la siguiente Tabla 3: 


\section{Tabla 3}

Subneteo de Red

\begin{tabular}{cccccc}
\hline $\mathrm{N}^{\circ}$ & \multicolumn{1}{c}{ Red } & \multicolumn{2}{c}{ Rango IP } & Broadcast & Host \\
\hline 01 & 192.168 .1 .0 & 192.168 .1 .1 & 192.168 .1 .14 & 192.168 .1 .15 & 14 \\
\hline 02 & 192.168 .1 .16 & 192.168 .1 .17 & 192.168 .1 .30 & 192.168 .1 .31 & 14 \\
\hline 03 & 192.168 .1 .32 & 192.168 .1 .33 & 192.168 .1 .46 & 192.168 .1 .47 & 14 \\
\hline 04 & 192.168 .1 .48 & 192.168 .1 .49 & 192.168 .1 .62 & 192.168 .1 .63 & 14 \\
\hline 05 & 192.168 .1 .64 & 192.168 .1 .65 & 192.168 .1 .78 & 192.168 .1 .79 & 14 \\
\hline 06 & 192.168 .1 .80 & 192.168 .1 .81 & 192.168 .1 .94 & 192.168 .1 .95 & 14 \\
\hline 07 & 192.168 .1 .96 & 192.168 .1 .97 & 192.168 .1 .110 & 192.168 .1 .111 & 14 \\
\hline 08 & 192.168 .1 .112 & 192.168 .1 .113 & 192.168 .1 .126 & 192.168 .1 .127 & 14 \\
\hline 09 & 192.168 .1 .128 & 192.168 .1 .129 & 192.168 .1 .142 & 192.168 .1 .143 & 14 \\
\hline 10 & 192.168 .1 .144 & 192.168 .1 .145 & 192.168 .1 .158 & 192.168 .1 .159 & 14 \\
\hline 11 & 192.168 .1 .160 & 192.168 .1 .161 & 192.168 .1 .174 & 192.168 .1 .175 & 14 \\
\hline 12 & 192.168 .1 .176 & 192.168 .1 .177 & 192.168 .1 .190 & 192.168 .1 .191 & 14 \\
\hline 13 & 192.168 .1 .192 & 192.168 .1 .193 & 192.168 .1 .206 & 192.168 .1 .207 & 14 \\
\hline 14 & 192.168 .1 .208 & 192.168 .1 .209 & 192.168 .1 .222 & 192.168 .1 .223 & 14 \\
\hline 15 & 192.168 .1 .224 & 192.168 .1 .225 & 192.168 .1 .238 & 192.168 .1 .239 & 14 \\
\hline 16 & 192.168 .1 .240 & 192.168 .1 .241 & 192.168 .1 .254 & 192.168 .1 .255 & 14 \\
\hline & & & & & \\
\hline
\end{tabular}

Después de haber subneteado la red, se van a obtener los siguientes datos que se muestran en la Tabla 4.

\section{Tabla 4}

Resultados del Subneteo

\begin{tabular}{ll}
\hline Red & $192.168 .1 . X$ \\
\hline Máscara & 255.255 .255 .240 \\
\hline Subredes & 5 para usar \\
\hline
\end{tabular}

Mientras que en la Tabla 5 se evidencian los datos de la distribución Vlans.

\section{Tabla 5}

Distribución de Vlans

\begin{tabular}{llll}
\hline Vlan & \multicolumn{1}{c}{ Nombre } & \multicolumn{1}{c}{ Puertos de Switch } & \multicolumn{1}{c}{ Modo Puertos } \\
\hline 1 & Admin & G1, G2, F21 - F24 & Modo Truncado G1 \\
\hline 10 & PC & F1-F5 & Modo Acceso \\
\hline 20 & Camaras & F6 - F9 & Modo Acceso \\
\hline 30 & Telefonía & F10 - F16 & Modo Acceso \\
\hline 40 & Access Point & F17 - F20 & Modo Acceso \\
\hline
\end{tabular}


Figura 2

Topología de la red

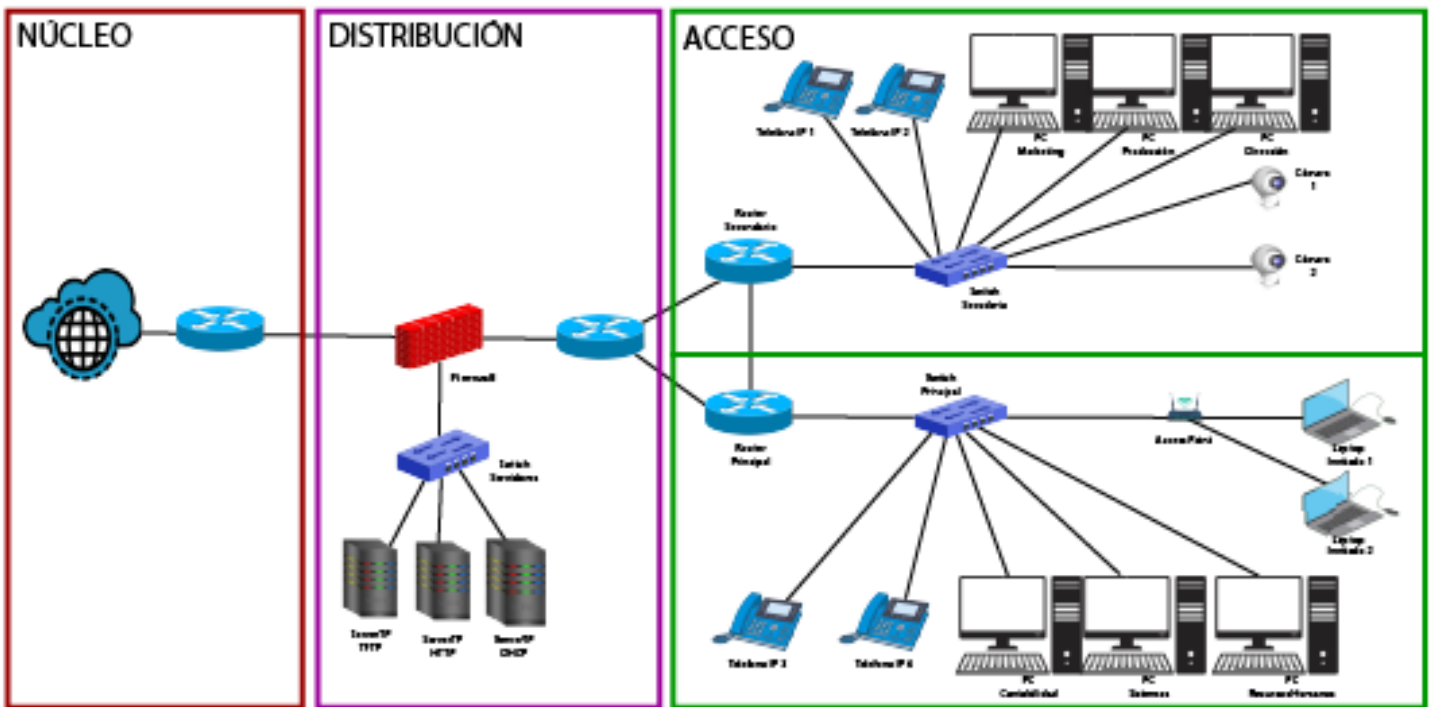

Antes de comenzar con las configuraciones, lo que vamos a realizar, es la búsqueda de cada uno de los objetos graficados en la topología (Figura 2), por lo cual, las herramientas que se escogieron para montar la red son:

- $\quad$ Servidores para HTTP, DHCP y TFTP con la herramienta Server TP. Los servidores, como se ve en la Figura 2, se encuentran en la capa de distribución.

- Switches, para las diferentes sucursales y los servidores, de tipo 2969-24TT. Los switches se encuentran en la capa de acceso.

- Router de tipo 1841, está en la capa de distribución, y otros dos routers se encuentran en la capa de acceso, al igual que uno se encuentra en la capa núcleo.

- Teléfonos IP, Pc’s, Laptops y Wireless se encuentran en la capa de acceso.

Después de graficar la topología, se plantea la configuración de los servidores, el servidor escogido permite configurar todo mediante interfaz gráfica, por lo cual simplemente se habilita o deshabilita HTTP, DHCP y TFTP (Figura 3). 


\section{Figura 3}

Configuración de los servidores TFTP, HTTP y DHCP

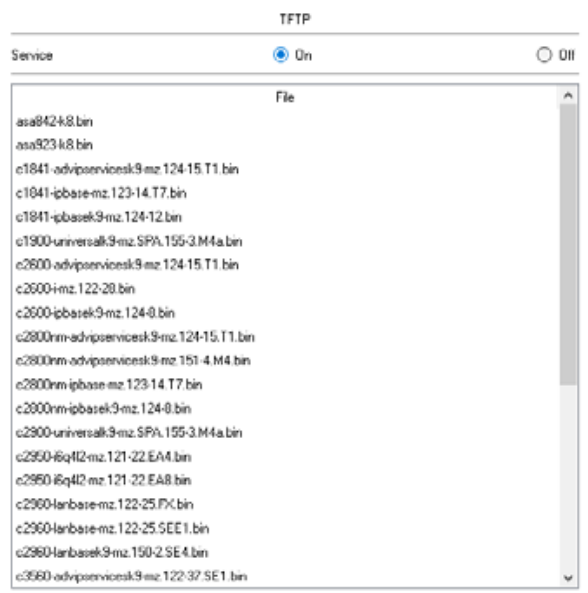

\begin{tabular}{l|l}
\hline \multicolumn{1}{l}{ HTTP } \\
\hline
\end{tabular}

\begin{tabular}{|c|c|c|c|c|c|c|c|c|}
\hline \multicolumn{9}{|c|}{$\mathrm{DHCP}$} \\
\hline nterlace & \multicolumn{2}{|c|}{ FactEthernet0 } & $\checkmark$ & \multicolumn{3}{|c|}{ Servce $\bigcirc$ Un } & \multicolumn{2}{|c|}{ (C) UH } \\
\hline \multicolumn{4}{|l|}{ Jool Name } & \multicolumn{5}{|c|}{ serverPool } \\
\hline \multicolumn{4}{|c|}{ Jofoulk Gotowoy } & \multicolumn{5}{|c|}{0.0 .00} \\
\hline \multicolumn{4}{|l|}{ JNS Server } & \multicolumn{5}{|c|}{0.0 .0 .0} \\
\hline \multicolumn{2}{|c|}{ Ŝlat IP Addiess : 192} & \multicolumn{2}{|c|}{168} & \multicolumn{3}{|c|}{1} & \multicolumn{2}{|l|}{0} \\
\hline Sิuhnet Mask: & 255 & 255 & & \multicolumn{3}{|c|}{$\mid 255$} & \multicolumn{2}{|l|}{240} \\
\hline \multicolumn{4}{|c|}{ Maximum Number of Users: } & \multicolumn{5}{|l|}{16} \\
\hline \multicolumn{4}{|l|}{ IFIP Server. } & \multicolumn{5}{|c|}{0.0 .0 .0} \\
\hline \multicolumn{4}{|l|}{ ML Address: } & \multicolumn{5}{|c|}{0.0 .0 .0} \\
\hline \multicolumn{2}{|c|}{ add } & \multicolumn{4}{|c|}{ Save } & \multicolumn{3}{|c|}{ Remove } \\
\hline $\begin{array}{l}\text { Poul } \\
\text { Namie }\end{array}$ & $\begin{array}{l}\text { Defoull } \\
\text { Godeway }\end{array}$ & $\begin{array}{l}\text { DNS } \\
\text { Selver }\end{array}$ & & & $\begin{array}{l}\text { Subret } \\
\text { Mask }\end{array}$ & $\begin{array}{l}\text { Max } \\
\text { Usel }\end{array}$ & $\begin{array}{l}\text { TFTP } \\
\text { Setvel }\end{array}$ & $\begin{array}{c}\text { WLC } \\
\text { B,duress }\end{array}$ \\
\hline serverPonl1 & 0000 & 0000 & 1921 & 68212 & 552552 & 16 & $n \cap 0 n$ & $0 \cap 00$ \\
\hline serverPuol & 0.0 .0 .0 & 0.0 .0 .0 & 192.1 & 68.1 .12 & 255.255 .24 & 16 & 0.0 .0 .0 & 0.0 .00 \\
\hline
\end{tabular}

Por consiguiente, se continua con la configuración del switch, esto se realiza mediante los siguientes comandos que se ven en la Tabla 6, Tabla 7, Tabla 8 y Figura 4. 


\section{Tabla 6}

\section{Configuración de Switch}

\begin{tabular}{|c|c|c|}
\hline Actividad & Descripción & Código \\
\hline Creación de Vlans & $\begin{array}{l}\text { Se crean las distintas } \\
\text { vlans para cada uno de los } \\
\text { dispositivos que se conectarán } \\
\text { a la red }\end{array}$ & $\begin{array}{l}\text { Switch\# config t } \\
\text { Switch(config)\# vlan } 10 \\
\text { Switch(config-vlan)\# name Computadoras } \\
\text { Switch(config-vlan)\# vlan } 20 \\
\text { Switch(config-vlan)\# name Camaras } \\
\text { Switch(config-vlan)\# vlan } 30 \\
\text { Switch(config-vlan)\# name Telefonía }\end{array}$ \\
\hline $\begin{array}{l}\text { Asignación de puertos y } \\
\text { modos }\end{array}$ & $\begin{array}{l}\text { Para cada una de las vlans } \\
\text { creadas se les asignará los } \\
\text { respectivos puertos a los } \\
\text { que se podran conectar. Se } \\
\text { precentan dos ejemplos de } \\
\text { esta configuración. }\end{array}$ & $\begin{array}{l}\text { •Vlan Computadoras } \\
\text { Switch(config)\# int rang fa0/1-5 } \\
\text { Switch(config-if-range)\# sw mo acc } \\
\text { Switch(config-if-range)\# sw acc vlan } 10 \\
\text { Switch(config-if-range)\# exit } \\
\text { •Vlan Camaras } \\
\text { Switch(config)\# int rang fa0/6-9 } \\
\text { Switch(config-if-range)\# sw mo acc } \\
\text { Switch(config-if-range)\# sw acc vlan } 20 \\
\text { Switch(config-if-range)\# exit }\end{array}$ \\
\hline $\begin{array}{l}\text { Asignación de modo } \\
\text { trunk }\end{array}$ & $\begin{array}{l}\text { Se asigna el modo trunk al } \\
\text { puerto gigabit para la conexión } \\
\text { con el router y se reflejen las } \\
\text { vlans. }\end{array}$ & $\begin{array}{l}\text { Switch(config0)\# int giO/1 } \\
\text { Switch(config-if)\# sw mo trunk } \\
\text { Switch(config-if)\# sw trunk allowed vlan } \\
1,10,20,30,40 \\
\text { Switch(config-if)\# end } \\
\text { Switch\# wr }\end{array}$ \\
\hline
\end{tabular}




\subsection{Configuración del Router}

Tabla 7

\section{Configuración del Router}

\begin{tabular}{|c|c|c|}
\hline Actividad & Descripción & Código \\
\hline $\begin{array}{l}\text { Encender puerto fa0/0 } \\
\text { que está conectado al } \\
\text { Switch }\end{array}$ & $\begin{array}{l}\text { Se eciende el puerto que } \\
\text { esta conectado al switch para } \\
\text { proceder con la configuración }\end{array}$ & $\begin{array}{l}\text { Router>enable } \\
\text { Router\# config t } \\
\text { Router(config)\#in fa 0/0 } \\
\text { Router(config-if)\#no shut } \\
\text { Router(config-if)\#exit } \\
\end{array}$ \\
\hline $\begin{array}{l}\text { Sub-interfaces para } \\
\text { enrutamiento inter - } \\
\text { VLAN }\end{array}$ & $\begin{array}{l}\text { Se asiganan a cada una de las } \\
\text { vlan creadas las ip de entrada } \\
\text { al igual que las mascaras. } \\
\text { A contiuación se observa el } \\
\text { ejemplo. }\end{array}$ & $\begin{array}{l}\text {-Vlan Admin } \\
\text { Router(config)\#int fa 0/0.1 } \\
\text { Router(config-subif)\#encapsulation dot1Q } 1 \\
\text { Router(config-subif)\#ip add 192.168.1.1 } \\
255.255 .255 .240 \\
\text { Router(config-subif)\#exit } \\
\text {-Vlan Computadoras } \\
\text { Router(config)\#int fa 0/0.10 } \\
\text { Router(config-subif)\#encapsulation dot1Q } 10 \\
\text { Router(config-subif)\#ip add 192.168.1.17 } \\
255.255 .255 .240 \\
\text { Router(config-subif)\#exit }\end{array}$ \\
\hline $\begin{array}{l}\text { Configuración de la } \\
\text { telefonía }\end{array}$ & $\begin{array}{l}\text { Se habilita el servicio de } \\
\text { telefonia en los routers, y la } \\
\text { cantidad de telefonos que } \\
\text { se habilitarán al igual que la } \\
\text { dirección ip de enlace. }\end{array}$ & $\begin{array}{l}\text { Router\# config t } \\
\text { Router(config)\#telephony-service } \\
\text { Router(config-telephony)\#max-dn } 10 \\
\text { Router(config-telephony)\#max-ephones } 10 \\
\text { Router(config-telephony)\#create cnf-files } \\
\text { Router(config-telephony)\#ip source-address } \\
\text { 192.168.1.49 port } 2000 \\
\text { Router(config-telephony)\#auto assign } 4 \text { to } 6 \\
\text { Router(config-telephony)\#auto assign } 1 \text { to } 5 \\
\text { Router(config-telephony)\#exit }\end{array}$ \\
\hline $\begin{array}{l}\text { Configuración número } \\
\text { o extensión para cada } \\
\text { teléfono. }\end{array}$ & $\begin{array}{l}\text { Se configura el numero de } \\
\text { telefono para cada uno de los } \\
\text { teléfonos que se conectarán a } \\
\text { la red. }\end{array}$ & $\begin{array}{l}\text { Router(config)\#telephony-service } \\
\text { Router(config-telephony)\#ephone-dn } 1 \\
\text { Router(config-ephone-dn)\#number } 301 \\
\text { Router(config-ephone-dn)\#exit } \\
\text { Router(config)\#ephone-dn } 2 \\
\text { Router(config-ephone-dn)\#number } 302 \\
\text { Router(config-ephone-dn)\#exit } \\
\text { Router(config)\#ephone-dn } 3 \\
\text { Router(config-ephone-dn)\#number } 303 \\
\text { Router(config-ephone-dn)\#exit } \\
\text { Router(config)\#end } \\
\text { Router\#wr }\end{array}$ \\
\hline
\end{tabular}




\section{Tabla 8}

\section{Configuración para la conexión entre routers}

\begin{tabular}{|c|c|c|}
\hline Actividad & Descripción & Código \\
\hline \multirow{6}{*}{$\begin{array}{l}\text { Habilitación de puertos } \\
\text { seriales }\end{array}$} & \multirow{6}{*}{$\begin{array}{l}\text { Se incian los puertos seriales } \\
\text { s0/1/1, el mismo proceso se } \\
\text { realiza en los dos routers. }\end{array}$} & Router(config)\#int s0/1/1 \\
\hline & & $\begin{array}{l}\text { Router(config-if)\#ip add 192.168.30.1 } \\
255.255 .255 .0\end{array}$ \\
\hline & & Router(config-if)\#clockrate 2000000 \\
\hline & & Router(config-if)\#no shut \\
\hline & & Router(config-if)\#exit \\
\hline & & Router(config)\#end \\
\hline \multirow{10}{*}{$\begin{array}{l}\text { Enrutamiento con } \\
\text { protocolo RIP }\end{array}$} & \multirow{10}{*}{$\begin{array}{l}\text { Se inicia el protocolo rip y se } \\
\text { asigna la versión, des pues se } \\
\text { asignan los enlaces. La misma } \\
\text { configuración se realiza en los } \\
\text { dos routers. }\end{array}$} & Router(config)\# router rip \\
\hline & & Router(config)\# version 2 \\
\hline & & Router(config)\# no auto-summary \\
\hline & & Router(config)\# network 192.168.3.09 \\
\hline & & Router(config)\#network 192.168.30.0 \\
\hline & & Router(config)\#network 192.168.3.49 \\
\hline & & Router(config)\#dial-peer voice 1 voip \\
\hline & & Router(config-dial-peer)\#destination-pattern $4 .$. \\
\hline & & $\begin{array}{l}\text { Router(config-dial-peer)\#session target } \\
\text { ipv4:192.168.4.49 }\end{array}$ \\
\hline & & Router(config)\#ip route $0.0 .0 .0 \quad 0.0 .0 .0 \mathrm{~s} 0 / 1 / 1$ \\
\hline
\end{tabular}


Figura 4

Configuración de wireless

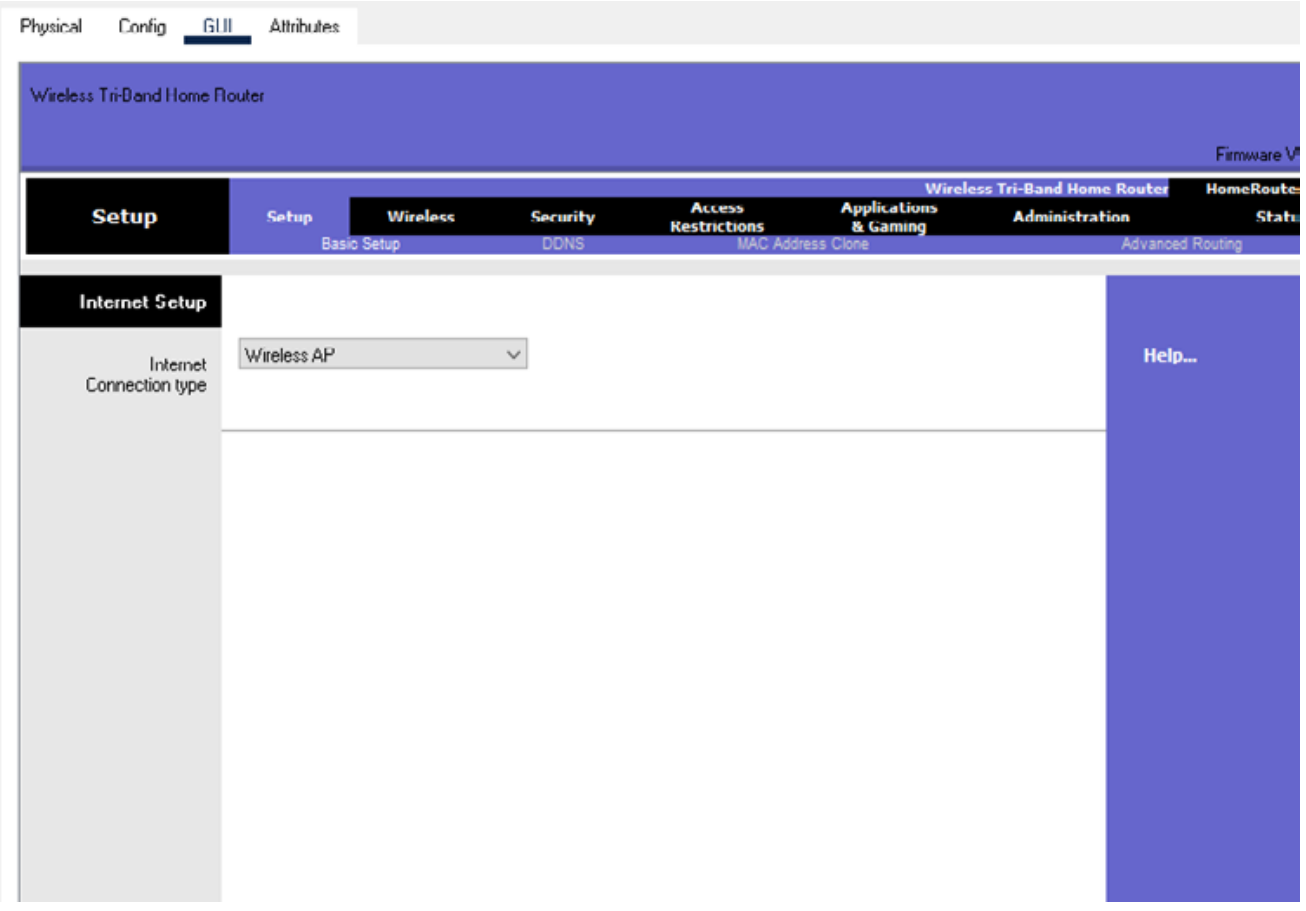

\section{Resultados}

Conforme a las configuraciones que se realizaron, se pone en funcionamiento la simulación de la red planteada, la misma que se observa en la Figura 5, en la cual se puede percibir que todos los dispositivos se encuentran conectados entre sí. Para verificar que los dispositivos están correctamente conectados lo que se realiza es un ping entre diferentes dispositivos, con el objetivo de mostrar que la red empresarial se encuentra en completo funcionamiento. 


\section{Figura 5}

Puesta en marcha de la red

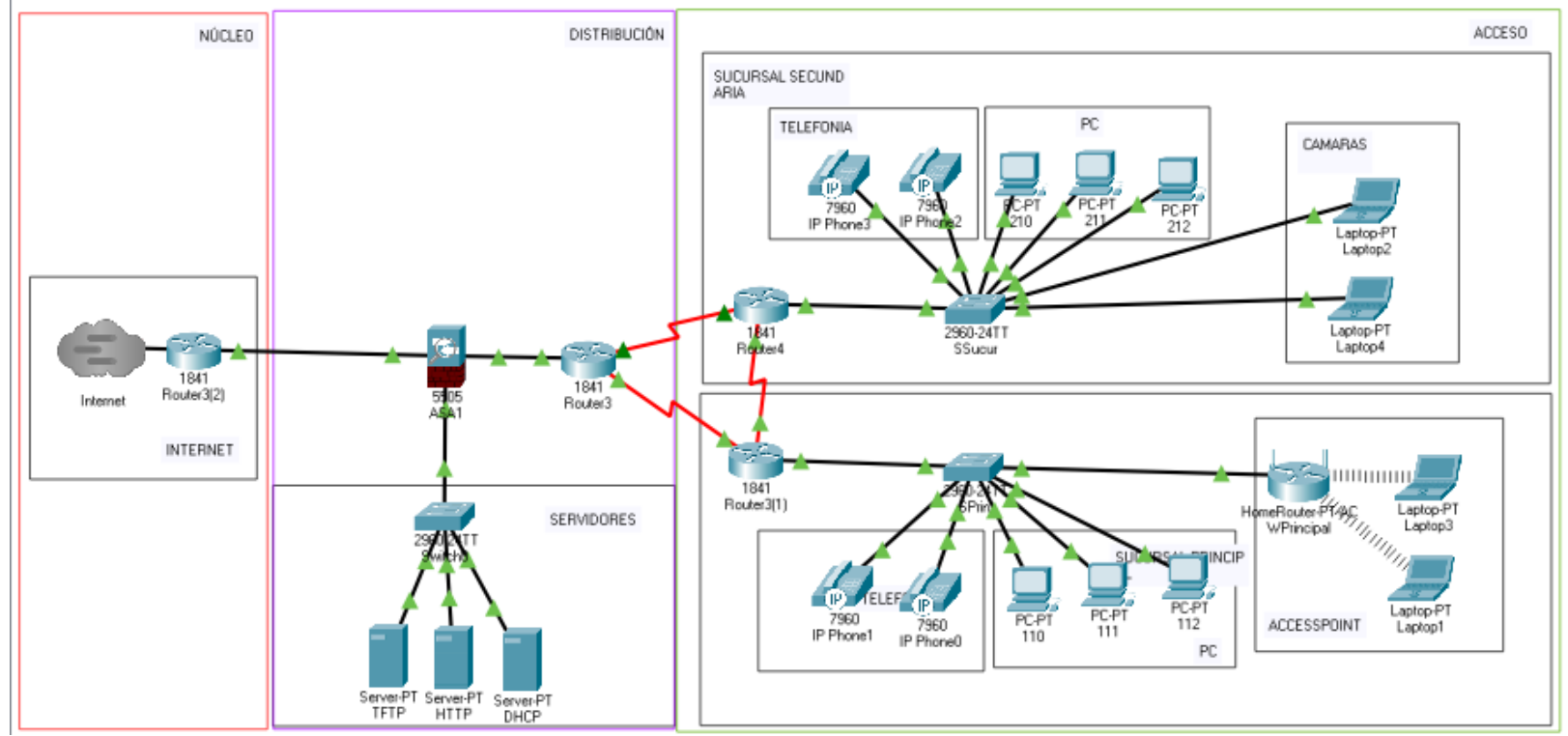

Por consiguiente, como se puede observar en la Figura 5, Cisco Packet Tracer permite dibujar o encerrar en diferentes secciones cada uno de los dispositivos según la capa en donde se encuentren.

\section{Figura 6}

Computador 192.168.1.12

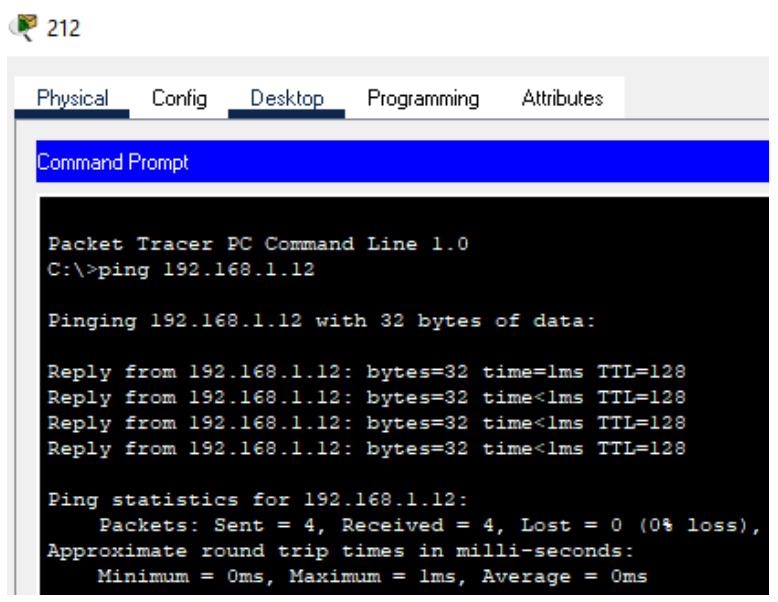

Figura 7

Computador 192.168.2.12

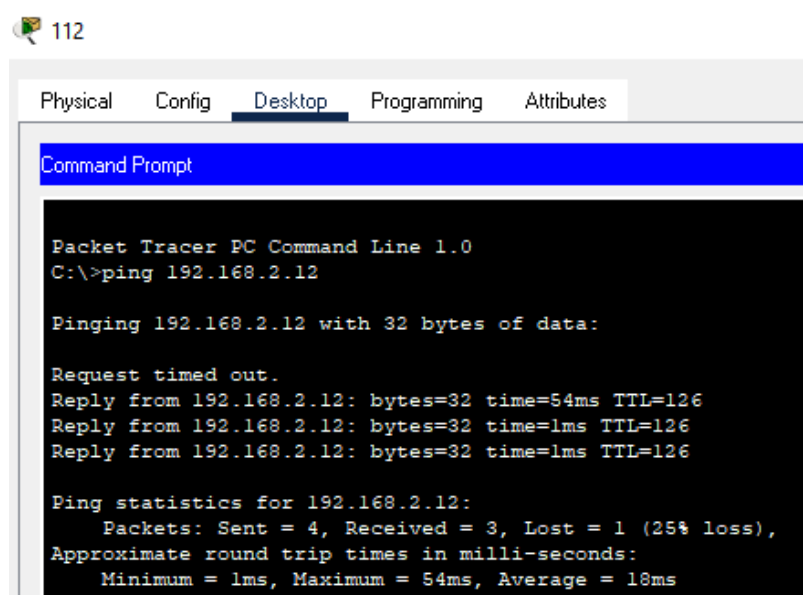

En las figuras anteriores se puede observar cómo si hay recepción y envío de paquetes, a través del comando ping, desde un computador que se encuentra en la sucursal principal (192.168.1.12), y una computadora de la sucursal secundaria (192.168.2.12). 
Figura 8

Ping desde Laptop3 a una Cámara

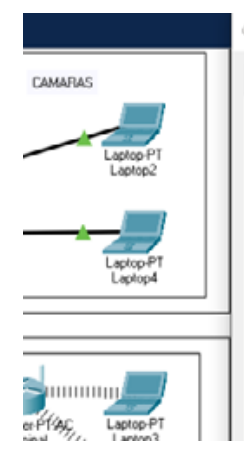

L Laptop3
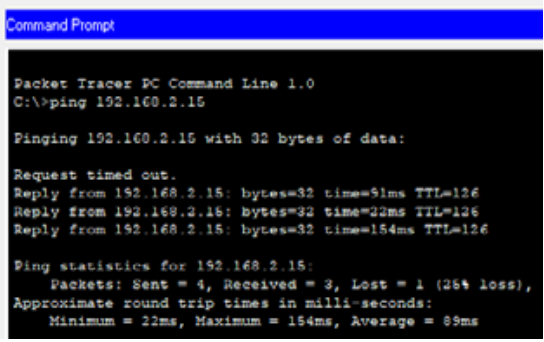

Figura 9

Ping hacia la laptop Wireless

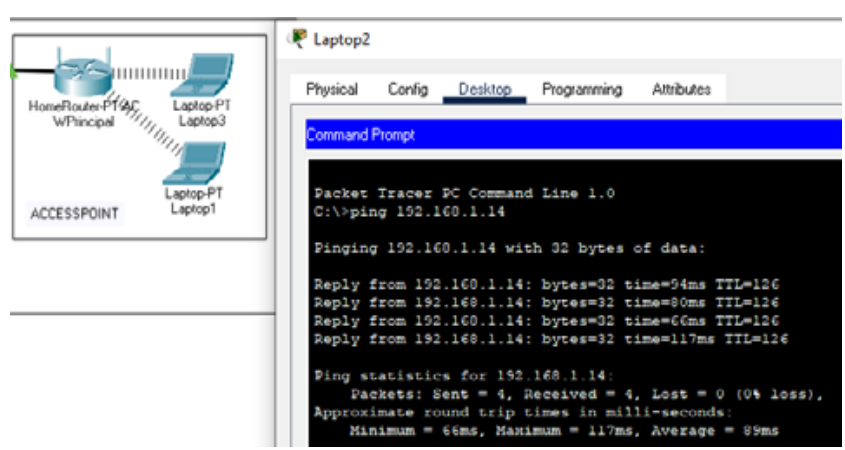

\section{Conclusiones}

Se puede concluir que se necesita primero definir correctamente el diseño que llevará la red, para una correcta organización de los dispositivos, al igual que la configuración de estos. El subneteo, como parte también de la organización de la red, permite la administración correcta de la red y la clasificación de esta.

La interconexión entre dispositivos es de gran importancia, ya que facilita la comunicación entre dispositivos y áreas de trabajo. Por lo cual, se puede disminuir algunos costos en la empresa, al igual que, proteger la información que comparten unas áreas con otras.

Algo muy importante que se logra ver dentro de toda la configuración es lo dinámica y sencilla qu resulta usar la herramienta Cisco Packet Tracer, que, aunque ya se solía utilizar antes de estar en pandemia, el uso de esta se ha duplicado, debido a la cantidad de dispositivos y herramientas que permite usar.

Finalmente, se puede decir que la herramienta antes del año 2019 llegaba a carecer de algunos dispositivos, pero las últimas actualizaciones han ido añadiendo más dispositivos con los cuales se puede interactuar, al igual que más funcionalidades y algunos ejemplos de conexión entre dispositivos. 


\section{Referencias}

Barragán Rojas, D. J., \& Jurado Solís, S. S. (2012). Aplicación del Simulador Packet Tracer para la realización de Prácticas en la Asignatura Telemática I de la Carrera de Ingeniería en Telecomunicaciones. [Tesis de grado, Universidad Católica de Santiago de Guayaquil] Repositorio Digital UCSG http://repositorio.ucsg. edu.ec/handle/3317/226

Bembibre, V. (2009). Router. DefinicionABC. https://www.definicionabc.com/tecnologia/router.php

Cámara, J. M. (2016). Infiniband: conceptos básicos: v. 2.0. https://riubu.ubu.es/bitstream/handle/10259/4230/ Fundamentos\%20de\%20Infiniband-1617.pdf?sequence $=1$

Cevallos, Y. (2010). Incidencia del uso del simulador de redes de computadoras packet tracer de cisco systems, en el desarrollo de competencias procedimentales, en la asignatura redes de computadoras II, del Cuarto Año de la Escuela de Sistemas, de la Facultad de Ingeniería, de la Universidad Nacional de Chimborazo, en el primer quimestre del periodo lectivo 2009-2010. [Tesis de maestría, Universidad Técnica de Ambato] Repositorio UTA. http://repositorio.uta.edu.ec/handle/123456789/5983

Citelia. (s.f). Qué es la telefonía ip y cómo funciona. https://citelia.es/blog/que-es-la-telefonia-ip-como-funciona/

De luz, S. (12 de agosto de 2021). VLANs: Qué son, tipos y para qué sirven. Redeszone. https://www.redeszone.net/tutoriales/redes-cable/vlan-tipos-configuracion/

Donoso, Y., Mendoza, P., \& Plata, N. (2001). Creación y especificación de subredes IP por medio de un enrutador con dos Puertos Lan. Ingeniería y Desarrollo, (9), 57-65. https://www.redalyc.org/pdf/852/85200905. pdf

Dordoigne, J. (2015). Redes informáticas-Nociones fundamentales. (Protocolos, Arquitecturas, Redes inalámbricas, Virtualización, Seguridad, IP v6...). Ediciones Eni.

Godoy Hernández, C. (2018). Diseño de una red Lan para el CDA Modifi-Car. [ Tesis de grado, Universidad Cooperativa de Colombia] Repositorio Institucional UCC. http://hdl.handle.net/20.500.12494/6328

Gómez, J., \& Pisco, C. (2018). Diseño e implementación de una red LAN jerárquica para la unidad educativa "Región Amazónica" [Tesis de grado, Universidad de Guayaquil] Repositorio UG http://repositorio.ug.edu. ec/handle/redug/33475

Huang, L. (2017). Diseño e Implementación de una red LAN para la empresa. [Tesis de grado, Universidad San Francisco de Quito] Repositorio USFQ.

Jesin, A. (2014). Packet Tracer Network Simulator. Packt Publishing Ltd. 
lawebdelprogramador. (s.f). Definición de Access Point https://www.lawebdelprogramador.com/diccionario/Access-Point/

Kurose, J. F., \& Ross, K. W. (2006). Redes de Computadores e a Internet. São Paulo: Person, 28.

Pérez Porto, J., \& Gardey, A. (2015). Definición.de Red Lan. Definición.de. https://definicion.de/red-lan/

Robleno, C. (1999). Redes de Computadoras. Editorial Ezime.

Significados. (31 de julio de 2017). Significado de Switch. https://www.significados.com/switch/ 


\section{Copyright (c) 2021 Carlos Alexander Chicaiza Puedmag}

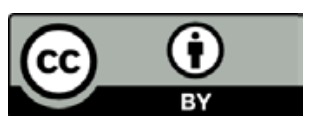

Este texto está protegido bajo una licencia internacional Creative Commons 4.0.

Usted es libre para Compartir-copiar y redistribuir el material en cualquier medio o formato - y Adaptar el documento - remezclar, transformar y crear a partir del material-para cualquier propósito, incluso para fines comerciales, siempre que cumpla las condiciones de Atribución. Usted debe dar crédito a la obra original de manera adecuada, proporcionar un enlace a la licencia, e indicar si se han realizado cambios. Puede hacerlo en cualquier forma razonable, pero no de forma tal que sugiera que tiene el apoyo del licenciante o lo recibe por el uso que hace de la obra.

\section{Resumen de licencia - Texto completo de la licencia}

